\title{
Peut-on rendre plus objective la détermination des débits réservés par une approche scientifique ?
}

\author{
How minimum instream flow determination can be more objective \\ with a scientific approach? \\ par Y. Souchon, S. Valentin et H. Capra \\ Cemagref Lyon \\ Laboratoire d'hydroécologie quantitative \\ Division Biologie des Ecosystèmes Aquatiques
}

The determination of minimum instream flow downstream from hydroelectric impoundments is composed of different aspects including social choices and technical evaluations. The available techniques, from purely hydrological methods to more complex biological models, are rapidly displayed to show that many helps in decision already exist. Besides, the relationships between hydrology and hydroecology are quite important now. The recurrent difficulty to obtain a consensus in the final decision lies more in the fact that the objectives are often not clear, and that the social evolutions towards ecological concerns are not well accepted. The result is that scientists are usually asked to improve again the methods instead of having the right debate. Among different solutions, the most important one is to obtain the essential feedback experience in ecological studies, with an accurate quantification of the real evolution of the stream after application of predicted minimum flows. It is the only new step to be expected, and it is the only one to avoid other laws like the "loi relative à la pêche en eau douce " of 1984, often regarded as too arbitrary.

\section{I INTRODUCTION}

Débit réservé, régime réservé, débit de référence biologique, débit biologique minimum acceptable, débit écologique : les termes pour désigner les débits à maintenir dans les cours d'eau à l'aval d'ouvrages de dérivation font florès. Cette débauche sémantique semble traduire la difficulté qu'éprouve la société à trouver un consensus sur les niveaux de protection souhaitables pour ces systèmes et à bâtir une police claire avec des objectifs eux-mêmes clairs et mesurables. Des logiques d'acteurs, souvent tendues par la défense d'un usage particulier, interfèrent dans ce débat, qui pourraient à elles seules faire l'objet d'un examen attentif, hors du champ de ce texte. Sollicité à son tour pour rendre plus objectif le débat, l'hydroécologiste propose des méthodes d'évaluation. Certaines d'entre elles sont significatives d'une évolution récente des modalités d'approche de l'écologie qui de descriptive et qualitative devient progressivement plus quantitative et prédictive. De même et de façon plus générale, on ne peut plus ignorer les relations existant entre l'hydrologie et les processus physiques et biologiques, qui participent au maintien de l'intégrité des cours d'eau et de leurs fonctionnalités. L'objectif de ce texte est de rappeler cette information disponible et immédiatement mobilisable sur les méthodes et les connaissances en hydroécologie. Nous analysons ensuite les facteurs de progrès envisageables au niveau des méthodes elles-mêmes.

\section{Q QUELS OBJECTIFS POUR LES COURS D'EAU ?}

C'est le point qu'il faut préciser en premier lieu avant toute application d'une quelconque méthode. Il deviendra alors plus simple de choisir les critères capables de prédire la réalisation de ces objectifs.

En France, la loi relative à la pêche en eau douce de 1984, puis la loi sur l'eau de 1992 mettent clairement l'accent sur la nécessité de considérer les cours d'eau comme un patrimoine commun de la nation, dont il faut assurer la pérennité de fonctionnement à long terme. On retrouve dans ces textes des préoccupations partagées au niveau international, en particulier depuis le sommet de Rio en 1992. Cette volonté du législateur de mieux équilibrer notre relation aux milieux, entre usages et préservation ne remporte pas, loin s'en faut, une adhésion générale. Elle est soit perçue comme une contrainte supplémentaire, soit carrément comme un manque à gagner par rapport à un usage prétabli souvent depuis très longtemps, comme la production d'hydroélectricité. Parmi 
les autres freins à une meilleure prise en compte de la préoccupation environnementale, figure en bonne place la difficulté de résumer les modalités de fonctionnement d'un hydrosystème en termes quantitatifs compréhensifs. La tentation est grande de vouloir disposer de critères quantifiés exprimant les demandes du milieu, de façon à les rendre facilement comparables à des critères économiques. Ce n'est sans doute pas un but inatteignable, encore faudrait-il y consacrer les moyens nécessaires et accepter ensuite les incertitudes inhérentes au fonctionnement de systèmes complexes aux processus rarement linéaires.

Il est bien évident qu'il faut s'abstraire de définitions trop vagues pour progresser. Avec un titre provocateur, " la qualité de l'eau : qualifier l'inqualifiable ", le livre de Boon et Howell [1] analyse très bien comment des notions mal définies dans le domaine voisin de la qualité de l'eau ne permettent pas d'agir efficacement. Ce n'est pas non plus avec des grandes sentences du type "les rivières doivent rester des rivières" [2] que l'on progressera.

Karr et Dudley [3] sont les premiers dans la littérature à avoir proposé une définition vraiment utile au débat en introduisant la notion d'intégrité biologique d'un cours d'eau, largement reprise depuis. Ils la définissent comme " la capacité d'un écosystème à supporter et maintenir une communauté d'organismes équilibrée, intégrée et adaptable avec une composition spécifique, une diversité, et une organisation fonctionnelle comparable à celle des habitats naturels d'une région ". Le cours d'eau est considéré dans toute sa complexité fonctionnelle et structurelle. Il est tenu compte de son statut régional potentiel, préoccupation que l'on retrouve actuellement en France dans la mise en place du Système d'Evaluation de la Qualité (SEQ chimique, biologique et physique des Agences de l'eau). Cette notion de référentiel est absolument indispensable pour mesurer l'écart entre situation actuelle et situation potentielle et apprécier les trajectoires possibles du fonctionnement du cours d'eau. Pour bien comprendre la notion de référence, peut-être est-ił besoin de prendre un exemple dans une branche disciplinaire voisine, la phytosociologie. On y bâtit des cartes de végétation potentielle en fonction des régions climatiques et géologiques, en imaginant des situations climaciques. En fonction de l'histoire de l'utilisation historique ou contemporaine des sols, la végétation réellement observable est plus ou moins proche de la situation de référence. Il est tout à fait possible d'adopter un même type de raisonnement pour la faune et la flore des cours d'eau.

On a trop souvent confondu ce besoin indispensable de référentiel avec la définition d'un objectif. L'objectif est un choix de trajectoire entre l'état actuel du cours d'eau et son potentiel : les débits négociés peuvent prendre différentes valeurs entre l'état actuel (on parlera alors de statu quo) et le référentiel naturel non influencé (on parle dans ce cas de restauration). Il doit bien sûr respecter les textes législatifs en vigueur. La loi sur l'eau a préféré porter la négociation entre usagers au plus près des acteurs organisés au sein de Communautés Locales de l'Eau ou CLE. Sans présager de leur fonctionnement futur, on entrevoit tout de même une difficulté probable à appliquer la loi dans le sens du bien commun au terme de ce type de négociation très locale entre usagers.

Plusieurs objectifs peuvent être retenus, parmi lesquels :

- des objectifs purement quantitatifs faisant plutôt appel à des critères physiques en termes de ressource quantitative, d'auto-entretien du lit de la rivière, voire de réhabilitation du caractère même du cours d'eau [4]. C'est une notion assez subjective, qui fait intervenir la largeur au miroir sur un ou plusieurs seuils : c'est ce type de critère qui a fini par prévaloir dans la décision de relèvement du débit réservé de $10 / 20 \mathrm{~m}^{3} / \mathrm{s}$ à $100 \mathrm{~m}^{3} / \mathrm{s}$, dans la portion court-circuitée du Rhône à Pierre-Bénite à l'aval immédiat de Lyon,

- des objectifs plus qualitatifs, en termes généraux de protection de l'environnement aquatique ou en termes plus sectoriels d'entretien de la ripisylve, de protection de l'habitat d'espèces patrimoniales à haute valeur symbolique (exemple du saumon atlantique à l'aval du barrage de Poutès Monistrol, rivière Allier [5]), d'espèces clés (truite fario, barbeau fluviatile,...) ou encore de l'ensemble des peuplements aquatiques.

On constate des changements majeurs de préoccupation au tournant des années 1990 tant en France que dans d'autres pays (ex. Angleterre [6]) : il faut rééquilibrer l'exploitation des ressources des écosystèmes avec leur maintien à long terme (durable). La notion de protection de la biodiversité pour les générations futures est aussi de plus en plus affirmée.

Ces objectifs pris isolément ou de façon combinée, vont nécessiter le recours à des méthodes soit essentiellement physiques (hydrologie et géométrie hydraulique) sans grande mobilisation des connaissances sur le fonctionnement des écosystèmes, soit plus écologiques centrées principalement sur la notion d'habitat.

\section{III — LES MÉTHODES}

\subsection{Approches hydrologiques}

Les valeurs qualifiant l'étiage des cours d'eau ont tout d'abord assez naturellement été choisies par les hydrologues auparavant confrontés à la définition de débits standards pour la dilution des eaux. C'est ainsi qu'ont été avancés le 7Q10 aux USA (débit le plus bas au cours de 7 jours consécutifs, à fréquence d'une fois seulement tous les dix ans), le Q 347 en Grande Bretagne et au Pays de Galles, équivalent au percentile $95 \%$ de la courbe des débits classés ou le QMNA 5 en France en vigueur pour les objectifs de qualité. Ces débits souvent très bas en fréquence et en valeurs absolues n'ont généralement pas été retenus pour satisfaire des objectifs de maintien des communautés aquatiques [7] et [8].

Lorsque l'on reste dans ce seul registre de l'hydrologie, c'est sans conteste la méthode de Tennant [9] proposée pour le Middle West des USA, qui a été la plus plébiscitée, y compris par la loi française. Les débits sont calculés en pourcentage du module moyen interannuel, valeur en général beaucoup plus fiable que celles d'étiage (C. Michel, com.pers.). Le 1/10ème du module interannuel est considéré comme un minimum minimorum et $30 \%$ de ce même module comme une valeur acceptable. Les débits conseillés sont saisonnalisés et il est prévu de garantir un débit de "nettoyage" des substrats en produisant une crue artificielle une fois par an égale à $200 \%$ du module. On peut simplement constater que l'application française a été minimaliste. A l'appui de cette remarque les valeurs suivantes ont été relevées dans la littérature :

- Canada, façade atlantique : $25 \%$ du module (cité en [8]),

- Espagne, pays basque [2] pour des cours d'eau à caractère torrentiel :

- cours d'eau de rang 1: $46 \%$ Qmoy ; 
- de rang $2: 38 \%$

- de rang 3 et $4: 33 \%$,

- Grande Bretagne, $16 \%$ pour les chalk streams [6].

\section{- 3.2 Approches de géométrie hydraulique}

On tient ici compte de la morphologie des cours d'eau. Par des mesures simples sur des transects, le principe consiste soit à préserver une partie du lit mouillé, soit à ménager une certaine hauteur minimum dans le lit des cours d'eau en privilégiant parfois des sections plus rapides de type radier.

Parmi elles citons :

- la méthode du périmètre mouillé [10] : il s'agit d'identifier un point d'inflexion sur le graphe d'évolution de ce périmètre mouillé en fonction du débit. Un test effectué par Tennant de cette fonction a conduit à montrer que pour une centaine de cours d'eau analysés, il existait un point d'inflexion aux alentours de la valeur de 1/10ème du module interannuel. Sous réserve d'approfondissement, cela laisserait supposer l'existence d'une propriété physique commune à plusieurs cours d'eau, ce qu'il conviendrait de nuancer vraisemblablement par région et rang.

- la méthode basque [2] qui préconise de maintenir au minimum $60 \%$ du périmètre mouillé,

- la recommandation en vigueur en Suisse avec le maintien d'au moins $20 \mathrm{~cm}$ de hauteur d'eau dans les cours d'eau. Cette dernière méthode est à rapprocher de certains autres critères de hauteur minimum sur les radiers les plus critiques pour leur franchissabilité par les poissons [11].

\subsection{Approches habitat}

En se focalisant sur l'habitat, espace physique nécessaire à la vie des organismes, les méthodes de cette catégorie abordent véritablement des considérations d'ordre biologique. Nous laissons volontairement de côté les méthodes globales d'habitat de type HQI (Habitat Quality Index, [12]) ou Habscore [13]. Ce type de méthode a surtout pour objectif d'estimer la biomasse de poissons, le plus souvent des salmonidés, en fonction de différents paramètres explicatifs dont le débit, considéré de façon très statique. Fausch et al. [14] en ont fait une critique très complète.

Les approches les plus répandues et les plus performantes peuvent être toutes considérées comme des clones plus ou moins élaborés de la méthode dite des microhabitats énoncée par Bovee et Milhous (PHABSIM, Physical Habitat Simulation, [15]) et dont on trouve les premiers fondements dans Waters [16]. Elles se situent à une échelle stationnelle, représentative d'un tronçon homogène de cours d'eau et par rapport aux précédentes elles nécessitent un certain effort d'investigation tant pour le recueil des données que pour leur analyse.

Le principe général consiste à décrire et modéliser pour la portion de cours d'eau étudiée la répartition en fonction du débit des paramètres hydrodynamiques majeurs de l'habitat que sont la hauteur d'eau, la vitesse du courant et le substrat. Ces caractéristiques physiques du cours d'eau sont ensuite confrontées aux exigences connues des espèces aquatiques et de leurs stades vitaux majeurs pour en déduire une expression d'habitabilité potentielle. Plusieurs supports d'analyse aident ensuite l'expert dans l'étude de la sensibilité du cours d'eau au débit : des cartographies stationnelles, le comportement physique de toute la station, de ses faciès morphodynamiques ou même de chacun des transects mesurés, et la possibilité de bien sérier tous les stades sensibles de la vie d'une espèce. Cette analyse doit bien sûr être resituée dans son contexte hydrologique. Cette démarche est désormais bien admise en France et est assez systématiquement utilisée dans les études d'impact, en particulier les études de renouvellement des concessions hydroélectriques. On trouvera des descriptions plus complètes de la méthode dans [17], [18], [19]. Un progiciel dénommé EVHA (pour EValuation de l'HAbitat, [20], [21]), mis au point avec le concours du ministère de l'Environnement, est diffusé par le Cemagref pour permettre ce type d'application.

Par rapport aux deux types de méthodes précédemment évoqués, la méthode des microhabitats apparaît comme beaucoup plus complète. Elle permet d'abord des analyses purement physiques à différents niveaux de détail, mais surtout aborde le domaine biologique. Un certain pragmatisme a consisté à travailler dans un premier temps avec des espèces de poissons cibles comme la truite fario, ce qui permet de traiter nombre de situations soumises à modification de débits (cours d'eau énergétiques). La base de modèles biologiques s'est progressivement enrichie portant à 25 espèces le nombre de poissons désormais analysable [22]. Il n'est pas interdit de penser que dans l'avenir, des développements permettront d'intégrer les macroinvertébrés [23], voire les macrophytes.

Parmi les facteurs de progrès encore nécessaires, figure avant tout la démonstration de son réalisme biologique dans diverses situations. On raisonne, en effet, sur des habitats potentiels et sur la notion de seuil d'accroissement du risque en dessous duquel cet habitat n'assurerait plus la survie autonome de l'espèce. Dans une négociation parfois serrée mêlant experts biologistes et non biologistes, il est parfois difficile de faire admettre certains principes écologiques de non linéarité des réponses aux modifications de débit. C'est ici que la discussion initiale sur la nécessité de bien s'entendre sur les objectifs prend toute sa signification : on trouve pêle-mêle des confusions entre habitat maximum, habitat optimum, biomasse maximale etc. Sans référence à un fonctionnement potentiel du cours d'eau et donc à une analyse de son contexte hydrodynamique et biologique régional, il est très difficile de conclure. Cela revient implicitement à reconnaître des fonctionnements différents et donc des sensibilités différentes pour différents types de cours d'eau. Plusieurs auteurs ([24], [25]) ont par exemple montré que les changements relatifs d'habitat potentiel étaient plus rapides et accentués dans les petits cours d'eau que dans les grands. Ce principe est transcrit d'une certaine façon dans les lois suisse et française.

Concernant le réalisme biologique de ces approches, c'està-dire la démonstration de liens entre potentiel d'habitat et populations ou peuplements aquatiques, il faut bien distinguer la mise en pratique du meilleur savoir mobilisable à un instant donné dans un processus de décision de la démarche de longue haleine, qui consiste à progresser dans la connaissance des mécanismes biologiques liés à l'hydrologie. Et surtout ne pas bloquer l'action en repoussant trop la prise de décision sous prétexte de connaissances à parfaire. Les progrès viendront désormais du suivi de situations pré- ou postmodification de débit. C'est dans cet esprit que travaillent désormais plusieurs équipes ; aux USA, suivi en cours sur 13 ans de 12 cours d'eau [26] et en France, les 7 cours d'eau de la cellule débits réservés [27].

Enfin, de gros progrès sont à attendre de travaux sur les peuplements dans leur globalité pour aborder les cours d'eau plus grands [28], [29]. 


\section{IV — PRINCIPES GOUVERNANT LE FONC- TIONNEMENT DES HYDROSYSTÈMES LOTIQUES ÉGALEMENT À PRENDRE EN COMPTE}

Dans l'esprit de la loi sur l'eau, avec une traduction effective dans les SDAGE, il y a une attente pour mieux formaliser les notions d'autosuffisance, de fonctionnement durable des hydrosystèmes. Cette idée est bien présente dans la littérature internationale sous le vocable de "sustaining river ecosystems". Il y a donc lieu de rappeler succinctement les quelques principes de base dont il sera de plus en plus tenu compte à l'avenir (voir aussi [30], [6], [31]).

Le maintien d'hydrosystèmes viables doit respecter les notions de :

- connectivité longitudinale et latérale (Amoros et Petts [32] mais aussi Brookes [33] pour qui toute action sur le physique peut avoir des répercussions sur les divers équilibres). En termes d'action, on fait par exemple appel à la notion d'espace de liberté des cours d'eau, et de transparence des ouvrages pour la migration des organismes,

- maintien de débits d'inondation pour la plaine alluviale, assurant différentes fonctions comme la dynamique de la matière organique et des sédiments fins, le maintien des ripisylves et des forêts alluviales, la recharge des nappes phréatiques, l'instauration de zones de reproduction saisonnières pour les poissons,

- maintien de débits d'entretien du lit (notion de crue morphogène) pour le rajeunissemnt des formes et l'entretien du substrat,

- débits "métronomes" sur lesquels sont calées les horloges biologiques (montaison, dévalaison des poissons, et divers autres rythmes biologiques).

Pour l'essentiel, nous avons retenu des fonctions liées à l'hydrologie. Elles devraient être mieux comprises, mieux vulgarisées et mieux quantifiées à l'avenir. Il subsiste donc tout un champ pour qualifier la notion de régime réservé qui s'imposera à terme.

\section{V $\square$ DISCUSSION. CONCLUSION}

A la lumière de ce qui vient d'être rappelé, on peut d'abord s'interroger sur l'efficacité du dispositif français. On a parfois perçu un jugement selon lequel la loi pêche était arbitraire, trop sévère ou trop simpliste. Par rapport à la situation régnant avant 1984, où beaucoup de débits réservés étaient nuls, elle a introduit un certain niveau de réhabilitation des cours d'eau en ménageant des distinctions entre situations existantes et situations nouvelles et une progressivité dans le temps. Il ne semble plus discutable que l'instauration systématique du 1/40ème du module ait été un réel progrès. Le passage au 1/10ème du module est une étape difficile à franchir, qui prend beaucoup plus de temps que ne l'avait prévu le législateur. Nous plaidons pour que les débats soient beaucoup plus francs autour d'objectifs explicités et quantifiés. Ce n'est qu'à cette condition que nous pourrons tirer tout le parti et la richesse des méthodologies qui ont vu le jour au cours des 15 dernières années. Il faut également préciser que l'analyse au cas par cas que permettent ces méthodes est complémentaire de l'existence des seuils légaux, bien pratiques par ailleurs pour les services instructeurs qui disposent ainsi de repères (communication au Conseil Général du GREF, Leynaud). En effet, le pétitionnaire doit bien apporter la preuve que le respect de ces seuils légaux de débit suffit pour satisfaire les articles premiers des lois pêche et eau qui ne sont que l'expression d'un regard d'une société qui évolue dans son rapport à l'environnement. A notre sens, la loi a donc été plutôt modérée et le chantier de la restauration et de la réhabilitation des hydrosystèmes est à peine ouvert.

Concernant les progrès méthodologiques, nous pensons qu'il ne faut pas forcément les attendre d'un trop grand raffinement des méthodes actuelles à résolution locale (au niveau des tronçons de cours d'eau), ciblées sur un nombre limité d'espèces. Il faut garder à l'esprit un certain réalisme pour leur applicabilité maximale et donc trouver un compromis acceptable en temps et en coût pour le recueil et le traitement des données. Les marges de progrès les plus probables viendront majoritairement des suivis de situations d'exploitation en vraie grandeur, où l'on aura fait varier les débits. Bien sûr, on ne fera pas l'économie d'études plus fonctionnelles sur les processus écologiques et la part du déterminisme hydrologique dans un but de parvenir à des modèles de prévision débits dépendant de l'intégrité des écosystèmes [6]. Cela conduira inévitablement à aborder la notion de régime réservé.

Enfin, on peut imaginer de possibles économies d'échelle si l'on devient capable de régionaliser les débits à réserver, ce qui nécessitera d'étoffer nos référentiels en hydrologie, en composition chimique des eaux, thermie, structure des corridors fluviaux, types de cours d'eau (forme du lit, séquences de faciès et ripisylve) et peuplements aquatiques.

\section{REMERCIEMENTS}

Les idées exprimées dans ce texte n'engagent que leurs auteurs. Elles s'inscrivent dans un débat permanent, passionné, mais toujours passionnant, conduit au fil du temps au sein de la cellule nationale débits réservés ou du club d'utilisateurs EVHA, avec différents interlocuteurs du ministère de l'Aménagement du Territoire et de l'Environnement et de ses services déconcentrés ou avec des aménageurs (EDF, CNR). Nous leur adressons nos remerciements collectifs.

\section{BIBLIOGRAPHIE}

[1] Boon, P.J. \& Howell, D.L. (1997). Freshwater quality : defining the indefinable? The Stationery Office, Edinburgh, $552 \mathrm{p}$.

[2] Docampo, L. \& De Bikuna, B.G. (1995). The basque metthod for determining instream flows in Northern Spain. Rivers, 4(4), 292-311.

[3] Karr, J.R. \& Dudley, D.R. (1981). Ecological perspective on water quality goals. Environmental Management, 5, 55-68.

[4] Jowett, I.G. (1997). Instream flow methods : a comparison of approaches. Regulated Rivers: Research \& Management, 13, 115-127.

[5] Valentin, S., Capra, H., Coton, T., Hérouin, E., Breil, P., Tholance, S. \& Souchon, Y. (1997). Simulation de l'habitat physique des salmonidés sur le Vieil-Allier, secteur de Poutès-Monistrol : aide à la détermination du débit. Cemagref BEA/LHQ - EDF-GEH Le Puy, 24 p.

[6] Petts, G.E. (1996). Water allocation to protect river ecosystems. Regulated rivers: Research \& management, 12, 353-365.

[7] Reiser, D.W., Wesche, T.A. \& Estes, C. (1989). Status of instream flow legislation and practices in North America. Fisheries, 14(2), 22-29.

[8] Dunbar, M., Gustard, A., Acreman, M. \& Elliott, C.R.N. (1997), Overseas approaches to setting river flow objectives. Technical Report, Env. Ag. Project W6B(96)4, 38p.

[9] Tennant, D.L. (1976). Instream flow regimes for fish, wildlife, recreation and related environmental resources, in Orsborn, J.F \& Allman, C.H. (Eds), Instream Flow Needs, American Fisheries Society, Western Division, Bethesda, Maryland, 359-373. 
[10] Cochnauer. T. (1976). Instream flow techniques for large rivers. in Orsborn. J.F. \& Allman, C.H. (Eds), Instream Flow Needs, American Fisheries Society, Western Division, Bethesda, Maryland, 387-399.

[1I] Nehring, R. (1979). Evaluation of instream flow methods and determination of water quantity needs for streams in the state of Colorado. Colorado Division of Wildlife, Fort Collins, 14-16-00006-78-909.

[12] Binns, N.A. \& Eisermann F.M. (1979). Quantification of fluvial trout habitat in Wyoming. Transactions of American Fisheries Society, 108(3). $215-228$

[13] Milner, N.J., Hemsworth, R.J. \& Jones, B.E. (1985). Habitat evaluation as a fisheries management tool. Journal of Fish Biology, 27, 85-108.

[14] Fausch. K.D., Hawkes, C.L. \& Parsons, M.G. (1988). Models that predict standing crop of stream fish from habitat variables : 1950-85. U.S Departement of Agriculture, Forest service, Pacific Northwest Research Station.. PNW-GTR-213

[15] Milhous, R.T. (1979). The PHABSIM system for instream flow studies. In Proceedings of the Summer Computer Simulation conf., Toronto, Ontario, Society for computer simulation, La Jolla, CA, 440-446.

[16] Waters, B.F. (1976). A methodology for evaluating the effects of differents streamflows on salmonid habitat. in Orsborn, J.F. \& Allman, C.H (Eds), Instream Flow Needs, American Fisheries Society, Western Division. Bethesda, Maryland, 254-266.

[17] Souchon, Y., Trocherie, F., Fragnoud, E. \& Lacombe, C. (1989). Les modèles numériques des microhabitats des poissons : application et nouveaux développements. Revue des Sciences de l'Eau, 2, 807-830.

[18] Pouilly, M., Valentin, S., Capra, H., Ginot, V. \& Souchon, Y. (1995). Note technique : Méthode des microhabitats, principes et protocoles d'application. Bulletin Français de Pêche et de Pisciculture, 336, 41-54.

[19] Sabaton, C., Valentin, S. \& Souchon, Y. (1995). La méthode des microhabitats, protocoles d'application. E.D.F Direction des Etudes et Recherches. Cemagref BEA/LHQ. HE-31/95/10, 33p.

[20] Ginot, V. (1995). EVHA : Un logiciel d'évaluation de l'habitat du poisson sous Windows. Bulletin Français de Pêche et de Pisciculture, 337. 338-339, 303-308,

[21] Ginot, V. \& Souchon, Y. (1995). Logiciel EVHA. Evaluation de l'habitat physique des poissons en rivière (version 1.0, beta test). Vol. I. Guide méthodologique. Vol. 2. Guide de l'utilisateur. Cemagref Lyon BEA/LHQ. France, et Ministère de l'Environnement, Dir. de l'Eau, Paris, France, 109p + annexes.
[22] Capra, H., Lamouroux, N., Valentin, S. \& Souchon, Y. (1997). Modélisation de l'habitat des poissons : définition d'une méthodologie évaluant l'impact de l'augmentation des débits réservés en système fluvial. CEMAGREF BEA/LHQ - CNR/Agence de I'Eau RMC, 28p.

[23] Wasson, J.G., Bonnard, R. \& Maridet, L. (1995). Réponses globales des invertébrés benthiques aux conditions d'habitat physique dans les cours d'eau salmonicoles : perspectives d'intégration dans les modèles habitat/poissons. Bulletin Français de Pêche et de Pisciculture, $337 / 338 / 339,291-299$

[24] Leonard, P.M. \& Orth, D.J. (1988). Use of habitat guilds of fishes to determine Instream Flow requirements. North American Journal of Fisheries Management, 8, 399-409.

[25] Jowett, I.G. (1992). Models of the abundance of large brown trout in New Zealand rivers. North American Journal of Fisheries Management, 12 , 417-432.

[26] Studley, T.D., Baldrige, J.E. \& Railsback, S.F. (1996). Predicting fish population response to instream flows. Hydro Review, 48-58.

[27] Merle, G. \& Eon, J. (1998). A full-scale test to validate the contribution of the IFIM procedure in the choice of a guaranteed flow downstream hydrostations, In Proceedings of the 2nd International Symposium on Habitat Hydraulics / 2e Symposium International sur l'Hydraulique et les Habitats, Québec, June / Juin 1996, INRS-Eau, FQSA, IAHR, 419-430.

[28] Lamouroux, N. (1997). Hydraulique statistique et prédiction de caractéristiques du peuplement piscicole : modèles pour l'écosystème fluvial. Thesis Doctorat, Université Claude Bernard Lyon I, Cemagref BEA LHQ.

[29] Capra, H. \& Souchon, Y. (1998). Peuplement de poisson de la Loire entre Grangent et Villerest. Cemagref/EDF, 26p.

[30] Souchon, Y. (1993). Hydrodynamique et écologie. Principes théoriques et modèles applicables à la gestion des cours d'eau à migrateurs. in (Eds), Colloque "Pour un retour des poissons migrateurs", Agence de I'Eau Adour- Garonne, 9-11 décembre 1993.

[31] Poff, N.L., Allan, J.D., Bain, M.B., Karr, J.R., Prestgaard, K.L., Richter, B.D., Sparks, R.E. \& Stromberg, J.C. (1997). The Natural Flow Regime. Bio Science, 47 (11), 769-784

[32] Amoros, C. \& Petts, G.E. (1993). Hydrosystèmes fluviaux. Paris $300 \mathrm{p}$

[33] Brookes, A. (1988). Channelized rivers. Perspectives for environnemental management. Wiley interscience, $326 \mathrm{p}$. 